\title{
Glycine Adsorption on Silicon (001)
}

\author{
A. C. Ferraz* and R. Miotto ${ }^{\dagger}$ \\ *Instituto de Física da Universidade de São Paulo, Caixa Postal 66318, CEP 05315-970, São Paulo, SP, Brazil \\ ${ }^{\dagger}$ Instituto de Física da Universidade de Brasília Caixa Postal 04455, CEP 70919-970, Brasília, DF, Brazil
}

Received on 4 April, 2005

\begin{abstract}
In this work we employ the state of the art pseudopotential method, within a generalized gradient approximation to the density functional theory, to investigate the dissociative adsorption process of glycine on the silicon surface. Our total energy calculations indicate that the chemisorption of the molecule is as follow. The gas phase $\mathrm{NH}_{2}-\mathrm{C}_{2} \mathrm{H}_{2}-\mathrm{OOH}$ adsorbs molecularly to the electrophilic surface $\mathrm{Si}$ atom and then dissociates into $\mathrm{NH}_{2}-\mathrm{C}_{2} \mathrm{H}_{2}-\mathrm{OO}$ and $\mathrm{H}$, bonded to the electrophilic and nucleophilic surface silicon dimer atoms respectively, with an energy barrier corresponding to a thermal activation that is smaller than the usual growth temperature, indicating that glycine molecules will be observed in their dissociated states at room temperature. This picture is further support by our calculated vibrational modes for the considered adsorbed species.
\end{abstract}

Keywords: Glycine adsorption; Silicon (001); Density functional theory

\section{INTRODUCTION}

In the past few years a great deal of effort has been devoted to the study of the adsorption and surface reaction mechanism of organic molecules on the silicon surface $[1,2]$. The progress achieved in the incorporation of organic functionality onto semiconductor surfaces was not only related to the understanding of its fundamental aspects, but also to its importance in various technological applications, such as: insulator films, resist of nanolithography, chemical and biological sensors, and molecular electronics. As expected, each of these needs requires the development of a well defined organic layer on the semiconductor surface. As such organic layers are basically formed by exposing the semiconductor surface to organic compounds, the understanding of the first stages of the interaction between the surface and the organic structure is crucial in order to improve the quality of deposited layers. In this sense, the capability of predicting the functionalization process of a number of different radicals will facilitate the selection, or design, of organic molecules which bond to the silicon (001) surface in a specific manner, and therefore the production of new devices. This understanding can be achieved by the analysis of model systems, such as amino acids, in detail. In particular, glycine has both the carboxylic acid and amine functionalities, making it an excellent prototype for the interaction of more complex organic molecules with the silicon surface. Indeed, the adsorption of glycine on clean silicon (001) surfaces has been the subject of a recent vibrational study by Lopez and co-workers [3]. Their high-resolution electron Energy loss spectroscopy (HREELS) indicates that the chemisorption of glycine occurs through cleavage of the $\mathrm{OH}$ bonds in the carboxylic group. In this work we employ the state of the art pseudopotential method, within a generalised gradient approximation to the density functional theory, to investigate the adsorption process of glycine on the silicon (001) surface. A detailed analysis of the structural properties of possible adsorbed systems are discussed and compared to available experimental results, with a view to contribute to further investigations, and to the understanding and interpretation of more complex systems including aspects of biochemically active thin films.

\section{THEORETICAL CONSIDERATIONS}

The surface was modelled in a super-cell geometry, with an atomic slab of six Si layers and a vacuum region equivalent to eight atomic layers. The unit cell for the $(2 \times 2)$ surface structure is spanned by vectors $\mathbf{a}_{1}=a(1,1,0)$ and $\mathbf{a}_{2}=a(-$ $1,1,0)$. The theoretical value of the bulk Si lattice constant $a$ was used in the surface calculations. On the top side of the slab we placed the glycine molecule in different configurations, and the back surface was passivated by $\mathrm{H}$ atoms arranged in a dihydride structure. Additionally, we made calculations for a single $\mathrm{NH}_{2}-\mathrm{C}_{2} \mathrm{H}_{2}-\mathrm{OOH}$ molecule placed in a cubic box of 22 atomic units (a.u.) side. The pseudopotentials for $\mathrm{Si}, \mathrm{C}, \mathrm{N}, \mathrm{O}$ and $\mathrm{H}$ were derived by using the scheme of Troullier and Martins [4] and the electron-electron exchange-correlation interactions were considered by using a generalised gradient approximation (GGA) [5] of the density functional theory. As for the surface calculations, the singleparticle orbitals were expressed in a plane-wave basis up to the kinetic energy of $35 \mathrm{Ry}$. For the Brillouin-zone summation, four special $\mathbf{k}$ points were used for surface calculations while for the isolated molecule we considered 8 special $\mathbf{k}$ points. Increasing the energy cut-off to $50 \mathrm{Ry}$ or the number of special k-points to 16 did not result in total energy differences by more than $0.1 \%$. The electronic and ionic degrees of freedom were relaxed by adopting the scheme described by Bockstedte et al [6]. The atoms were assumed to be in their fully relaxed positions when the forces acting on the ions were smaller than $0.005 \mathrm{eV} / \AA$. The most probable pathways and predicted energies from the gas-phase molecule to the adsorbed states were obtained by using a constraint dynamics scheme in which atomic movement along the pathway is a response to forces in a local region around it. The constraint is set only for the carbon (or hydrogen) atoms during the adsorption and dissociation processes. The energetic barriers are estimated following the phenomenological approach in the form of the Arrhenius equation [7] (for a more com- 
plete description see Ref. [9]). The adsorption energy for the glycine molecule was calculated as $E_{\text {conf }}^{a d s}=-\left[E_{\text {conf }}-E_{r e f}\right]$, where $E_{\text {conf }}$ is the total energy for the considered configuration, $E_{r e f}$ is the total energy for the free $\mathrm{Si}(001)-(2 \times 2)$ surface plus the total energy for a free glycine molecule in a box of side 22 a.u.. The relaxed adsorption geometries were used to calculate the zone-centre vibrational modes within the frozenphonon scheme [8]. For setting up the dynamical problem we considered the adsorbed system (the glycine molecule) and the Si dimer atoms.

\section{PRELIMINARY RESULTS}

For bulk silicon our first-principles calculations produced $5.42 \AA$ for the equilibrium lattice constant $\left(a_{o}\right), 0.86 \mathrm{GPa}$ for the bulk modulus $(B M)$, and $4.39 \mathrm{eV}$ for the cohesive energy, all in good agreement with the the experimental values presented in Ref. [10]. The calculated theoretical lattice constant obtained for the bulk silicon is used in surface calculations. The clean $\mathrm{Si}(001)-(2 \times 2)$ surface is characterised by alternated tilted $\mathrm{Si}-\mathrm{Si}$ dimers, i.e. one dimer component is at a higher position than the other, but neighbouring dimers present opposing tilting directions. The tilting of the dimer allows charge transfer from the 'down' atom (which becomes more planar or $s p^{2}$ bonded) to the 'up' atom (which becomes nearly pyramidal or $s^{2} p^{3}$ bonded). Thus, the 'down' Si atom dimer is electron deficient, while the 'up' Si atom is electron rich. Our calculations support this model: the $\mathrm{Si}-\mathrm{Si}$ dimer is found to have a bond length of $2.30 \AA$ and a vertical buckling of $0.73 \AA$, indicating a tilt angle of $17.8^{\circ}$. To test the reliability of the carbon, nitrogen, and hydrogen pseudopotentials, we performed additional calculations. For cubic diamond we obtained 3.60 (3.57) $\AA$, 4.53 (4.42) GPa and 7.28 (7.37) $\mathrm{eV}$ for the cubic lattice constant, bulk modulus, and cohesive energy, respectively, in good agreement with the experimental [10] values given in the parenthesis. Furthermore, our calculated bond lengths for the glycine molecule are $\mathrm{C}-\mathrm{C}=1.58 \AA, \mathrm{C}-\mathrm{N}=1.47 \AA, \mathrm{N}-\mathrm{H}=1.03 \AA, \mathrm{C}-\mathrm{O}=1.33 \AA$, $\mathrm{C}=\mathrm{O}=1.37 \AA, \mathrm{O}-\mathrm{H}=1.07 \AA$, and $\mathrm{C}-\mathrm{H}=1.11 \AA$, in good agreement with available experimental data [10].

\section{IV. $\mathrm{C}_{2} \mathrm{H}_{5} \mathrm{NO}: \mathrm{SI}(2 \times 2)-(001)$ SURFACE}

It is well established that the electron deficient (down) and electron rich (up) $\mathrm{Si}$ dimer atoms tend to react as electrophile and nucleaphile, respectively. In general, the dissociative adsorption of electron-rich species (Lewis bases), such as $\mathrm{H}_{2} \mathrm{O}$, is initiated by interaction with the electrophilic surface atom. As the $\mathrm{NH}_{2}-\mathrm{C}_{2} \mathrm{H}_{2}-\mathrm{OOH}$ molecule behaves like a Lewis bases, we expect it to initially adsorb on the electrophilic surface atom. Indeed we observe that glycine is adsorbed in a locally stable state, which then dissociate in $\mathrm{NH}_{2}-\mathrm{C}_{2} \mathrm{H}_{2}-\mathrm{OO}$ and $\mathrm{H}$, with the $\mathrm{H}$ component bonded to the nucleophilic surface atom. These observations are in total agreement with the dissociative adsorption model proposed by Lopez and co-workers [3] for the chemisorption of glycine on the silicon surface.
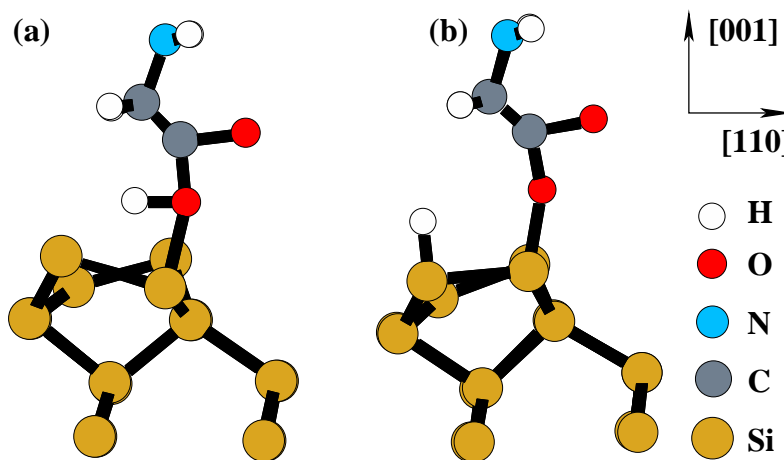

FIG. 1. Schematic views of the (a) molecularly (i.e. $\mathrm{NH}_{2}-\mathrm{C}_{2} \mathrm{H}_{2}-$ $\mathrm{OOH}$ bonded at one side of the $\mathrm{Si}$ dimmer) and dissociative (i.e. $\mathrm{NH}_{2}-\mathrm{C}_{2} \mathrm{H}_{2}-\mathrm{OO}$ and $\mathrm{H}$ bonded to different components of the dimmer) adsorption models of glycine on $\mathrm{Si}(001)-(2 \times 2)$.

In the following discussion, we are first going to address the equilibrium geometries for the glycine adsorbed species on the silicon surfaces in its molecular (i.e. $\mathrm{NH}_{2}-\mathrm{C}_{2} \mathrm{H}_{2}-$ $\mathrm{OOH}$ bonded at one side of the $\mathrm{Si}$ dimer as in Fig. 1 (a)) and dissociative (i.e. $\mathrm{NH}_{2}-\mathrm{C}_{2} \mathrm{H}_{2}-\mathrm{OO}$ and $\mathrm{H}$ bonded to different components of the dimer as in Fig. 1 (b)) states, and then the chemisorption energetics. Our first-principles calculations predict an almost symmetric $\mathrm{Si}-\mathrm{Si}$ dimer with a buckling of only $0.5^{\circ}$ for the dissociatively case and an asymmetric dimer with a buckling of $13^{\circ}$ for the molecularly adsorbed system. When the molecularly and dissociatively adsorbed systems are compared, we observe that the $\mathrm{Si}-\mathrm{Si}$ dimer gets more elongated for the later than for the former. In our present calculations, the dimer is elongated by $6 \%$ for the dissociative case, but only $3 \%$ for the molecularly adsorbed case. This suggests that the $\mathrm{Si}-\mathrm{O}$ bond is much stronger after the precursor dissociation occurs. This is further supported by the fact that the $\mathrm{Si}-\mathrm{O}$ bond length is around $0.1 \AA$ smaller for the dissociative adsorption model when compared to the molecular precursor state. Our data also suggests that, in general, both the $\mathrm{NH}_{2}-\mathrm{C}_{2} \mathrm{H}_{2}-\mathrm{OOH}$ and $\mathrm{NH}_{2}-\mathrm{C}_{2} \mathrm{H}_{2}-\mathrm{OO}$ adsorbed species retain the molecular gas phase characteristics, i.e. bond angle and bond lengths.

Our calculations suggest that the adsorption of the glycine molecule at the electrophilic surface (down) atom is an exothermic process, with an adsorption energy of $36 \mathrm{kcal} / \mathrm{mol}$. Our constraint dynamics scheme suggests that this adsorption process involves an energy barrier around $18 \mathrm{kcal} / \mathrm{mol}$. Considering the phenomenological approach in the form of the Arrhenius equation [7], with the choice for the A factor between $10^{13}-10^{15} \mathrm{~s}^{-1}$, we have estimated that, for complete dissociation (i.e., for an unitary reaction rate), an activation barrier of $18 \mathrm{kcal} / \mathrm{mol}$ corresponds to a thermal activation energy around $271 \mathrm{~K}$. The molecularly adsorbed $\mathrm{NH}_{2}-\mathrm{C}_{2} \mathrm{H}_{2}-\mathrm{OOH}$ dissociates, then, in $\mathrm{NH}_{2}-\mathrm{C}_{2} \mathrm{H}_{2}-\mathrm{OO}$ and $\mathrm{H}$, bonded respectively to the electrophilic and nucleophilic surface $\mathrm{Si}-\mathrm{Si}$ dimer atoms, with an energy gain of $41 \mathrm{kcal} / \mathrm{mol}$ when compared to the precursor molecular adsorbed state. The corresponding dissociation reaction presents a barrier of $24 \mathrm{kcal} / \mathrm{mol}$. This energy 
TABLE I: Comparison of the key structural parameters for the free glycine molecule (glycine ${ }_{\text {gas }}$ ), and the molecular and dissociative adsorption models of glycine on the $\mathrm{Si}(001)-(2 \times 2)$ surface. All bond lengths are in $\AA$, while bond angles are in degrees. Si-Si represents the $\mathrm{Si}-\mathrm{Si}$ dimer length for the adsorption site when the other dimer is unreacted.

\begin{tabular}{lccc}
\hline \hline bond length & glycine & gas & molecular \\
Si-Si & & 2.39 & 2.45 \\
$\mathrm{Si}-\mathrm{O}$ & & 1.84 & 1.73 \\
$\mathrm{Si}-\mathrm{H}$ & & & 1.49 \\
$\mathrm{O}-\mathrm{C}$ & 1.33 & 1.64 & 1.34 \\
$\mathrm{O}-\mathrm{H}$ & 1.07 & 1.06 & \\
$\mathrm{O}=\mathrm{C}$ & 1.19 & 1.17 & 1.21 \\
$\mathrm{C}-\mathrm{C}$ & 1.58 & 1.53 & 1.55 \\
$\mathrm{C}-\mathrm{N}$ & 1.47 & 1.40 & 1.44 \\
$\mathrm{C}-\mathrm{H}$ & 1.11 & 1.10 & 1.10 \\
$\mathrm{~N}-\mathrm{H}$ & 1.03 & 1.02 & $1 ; 03$ \\
bond angles & & 13 & 0.5 \\
$\mathrm{Si}-\mathrm{Si}$ & & 13 & 0.5 \\
$\mathrm{Si}-\mathrm{Si}-\mathrm{O}$ & & 95 & 119 \\
$\mathrm{Si}-\mathrm{Si}-\mathrm{H}$ & & & 117 \\
$\mathrm{H}-\mathrm{O}-\mathrm{C}$ & 105 & & \\
$\mathrm{O}-\mathrm{C}=\mathrm{O}$ & 119 & 116 & 123 \\
$\mathrm{O}-\mathrm{C}-\mathrm{C}$ & 113 & 116 & 116 \\
$\mathrm{C}-\mathrm{C}-\mathrm{N}$ & 124 & 126 & 123 \\
$\mathrm{H}-\mathrm{C}-\mathrm{H}$ & 105 & 104 & 104 \\
$\mathrm{H}-\mathrm{N}-\mathrm{H}$ & 107 & 108 & 107 \\
\hline \hline
\end{tabular}

barrier indicates that a thermal activation would lead the system into the dissociative chemisorption state. We have estimated that, for complete dissociation, an activation barrier of $24 \mathrm{kcal} / \mathrm{mol}$ corresponds to a thermal activation around $357 \mathrm{~K}$. This is consistent with the fact that Lopez and co- workers [3] have only observed the $\mathrm{NH}_{2}-\mathrm{C}_{2} \mathrm{H}_{2}-\mathrm{OO}$ at room temperature.

In the next step of our study, we have studied the dynamical properties of both the molecular and dissociative adsorption models in order to establish each of them corresponds to the structure experimentally observed by Lopez and coworkers [3]. For calculating the zone-centre optical phonon modes we set up a $66 \times 66$ eigenvalue problem, and identified modes that describe pronounced surface character. The results of our calculations as well as available experimental data, for stretch and bending modes for the molecular and dissociative adsorption models are presented in Table II. In general, our results for the dissociative adsorption model are consistent with the observations by Lopez and co-workers [3] whose experimental data also suggest that this is probably the adsorbed species observed. As expected, for the majority of the calculated surface modes, the differences between the molecular and dissociative vibrational spectra are not relevant. The main difference between the two models (that might be detectable in a HREELS experiment) is the $\mathrm{Si}-\mathrm{H}$ and $\mathrm{Si}-\mathrm{O}$ stretch modes. The Si-O stretch mode is found to be $767 \mathrm{~cm}^{-1}$ and $610 \mathrm{~cm}^{-1}$ for the dissociative and molecular adsorbed structures respectively. The $\mathrm{Si}-\mathrm{H}$ stretch mode of $2059 \mathrm{~cm}^{-1}$, on the other
TABLE II: Calculated vibrational modes for the dissociative and molecular adsorption models for the interaction of glycine with the $\mathrm{Si}(001)-(2 \times 2)$. All values are in $\mathrm{cm}^{-1}$, and the indicated symbols for the vibrationals are $s=$ symmetric, $a s=$ antisymmetric, $s t=$ stretch, scis=scissors.

\begin{tabular}{lccc}
\hline \hline \multicolumn{4}{c}{ adsorbed structure } \\
assignment & molecular & dissociative & exp. [3] \\
$\left(\mathrm{NH}_{2}\right)$ s st & 3534 & 3517 & 3460 \\
$\left(\mathrm{NH}_{2}\right)$ a st & 3432 & 3429 & 3410 \\
$\left(\mathrm{CH}_{2}\right)$ s st & 3120 & 3054 & 3040 \\
$\left(\mathrm{CH}_{2}\right)$ a st & 2980 & 3014 & 2940 \\
$\mathrm{Si}-\mathrm{H}$ st & & 2059 & 2115 \\
$\mathrm{C}=\mathrm{O}$ st & 1760 & 1724 & 1750 \\
$\left(\mathrm{NH}_{2}\right)$ scis & 1545 & 1594 & 1620 \\
$\left(\mathrm{CH}_{2}\right)$ scis & 1395 & 1383 & 1380 \\
$\mathrm{C}-\mathrm{O}$ st & 1016 & 1146 & 1170 \\
$\mathrm{C}=\mathrm{O}$ bend & 893 & 949 & 925 \\
\hline \hline
\end{tabular}

hand, is only observed for the dissociative adsorption model. Our calculated values for the dissociative adsorption model are consistent with the experimental values obtained by Lopez and co-workers [3] for the $\mathrm{Si}-\mathrm{H}$ and $\mathrm{Si}-\mathrm{O}$ stretch modes (2115 and $780 \mathrm{~cm}-1$, respectively). As HREELS is one of the most accurate surface science techniques for the identification of the bonding nature, , we believe that the experimental observations by Lopez and co-workers [3] combined with our firstprinciples calculations clearly indicate that the the dissociative adsorption model is most likely to be the adsorbed structure.

\section{SUMMARY}

We have performed first-principles calculations for the atomic structure and energetics of the adsorption of glycine on the silicon surface. Our data suggests that the global behaviour for the chemisorption of the glycine on the silicon surface is as follow: the gas phase $\mathrm{NH}_{2}-\mathrm{C}_{2} \mathrm{H}_{2}-\mathrm{OOH}$ adsorbs molecularly to the electrophilic surface $\mathrm{Si}$ atom and than dissociates into $\mathrm{NH}_{2}-\mathrm{C}_{2} \mathrm{H}_{2}-\mathrm{OO}+\mathrm{H}$, bonded to the electrophilic and nucleophilic surface silicon dimer atoms respectively, with an energy barrier corresponding to a thermal activation that is smaller than the usual growth temperature. This clear indicates that glycine will be observed in its dissociated states at room temperature in agreement with the findings by Lopez and co-workers [3]. This picture is further supported by our vibrational mode analysis. The dissociative adsorbed state is found to be $77 \mathrm{kcal} / \mathrm{mol}$ more stable than the system composed by the molecular gas phase and the free silicon surface. Both the molecularly and dissociated adsorbed systems are characterised by elongated $\mathrm{Si}-\mathrm{Si}$ dimers that are (almost) symmetric in the dissociative case but asymmetric in the molecular case. According to our first-principles calculations, the $\mathrm{NH}_{2}-\mathrm{C}_{2} \mathrm{H}_{2}-\mathrm{OOH}$ and $\mathrm{NH}_{2}-\mathrm{C}_{2} \mathrm{H}_{2}-\mathrm{OO}$ adsorbates retain the geometry observed for the gas phase glycine molecule. 
[1] R. J. Hamers, Y. Wang, and J. Shan, Appl. Surf. Science 107, 25 (1996).

[2] R. J. Hamers, J. S. Hovis, C. M. Greenlief, and D. F. Padowitz, Jpn. J. Appl. Phys. 38, 3879 (1999).

[3] A. Lopez, T. Heller, T. Bitzer, and N. V. Richardson Chem. Phys. 277, 1 (2002).

[4] N. Troullier and J. L. Martins, Phys. Rev. B 43, 1993 (1991).

[5] J. P. Perdew, J. A. Chevary, S. H. Vosko, K. A. Jackson, M. R. Pederson, D.J. Sing and C. Fiolhais, Phys.Rev. B 46, 6671 (1992).

[6] M. Bockstedte, A. Kley, J. Neugebauer, and M. Scheffler,
Comp. Phys. Commun. 107, 187 (1997).

[7] M. J. Pilling and P. W. Seakins, Reaction Kinetics (Oxford Science Publications, Oxford, 1995).

[8] G. P. Srivastava, The Physics of Phonons (Adam Hilger, Bristol, 1990).

[9] R. Miotto, G. P. Srivastava, and A. C. Ferraz, Phys. Rev. B 63, 125321 (2001).

[10] D. R. Lide, Handbook of Chemistry and Physics (Chemical Rubber Company, Roca Raton, 1995). 